\title{
AN APPLICATION OF GROUP THEORY TO HYDRODYNAMICS*
}

\author{
$\mathrm{BY}$
}

\section{E. J. WILCZYNSKI}

It has been observed by SopHus LiE that the stationary motion of a fluid can serve as a perfect picture of a one-parameter group in three variables. So far as I know, neither he nor any of his followers utilized this fact for the purposes of hydrodynamics. It is the purpose of the present paper to do this. One of the advantages gained for hydrodynamics by this standpoint lies in the general conception. But another advantage is, as is always the case when a class of problems is investigated from a new standpoint, that from the group-theoretical point of view, certain special cases are of exceptional interest, simplicity, and importance, cases which otherwise would appear difficult and unpromising.

\section{\$1. Relation between the steady motion of a fuid aud the theory of one-parameter groups.}

From the definition of the steady flow of a fluid, and LIE's conception of a oneparameter group, the following theorems will be seen to be true.

1. Let $x, y, \approx$ be the Cartesian coördinates of any point of the fluid at the time $t$, and let $a, b, c$ be the coördinates of the same material point at the time $t=0$. If

$$
x=\phi(a, b, c ; t), \quad y=\psi(a, b, c ; t), z=\chi(a, b, c ; t)
$$

are the equations which describe the steady motion of any point of the fluid, they are also the finite equations of a one-parameter group, the parameter being $t$.

2. Conversely, if equations (1) are the equations of a one-parameter group, they can also be interpreted as describing the steady flow of a fluid.

We shall be mainly concerned with the second theorem. _Let

$$
\mathrm{K} f=u \frac{\partial f}{\partial x}+v \frac{\partial f}{\partial y}+w \frac{\partial f}{\partial z}
$$

be the infinitesimal transformation of a one-parameter group $G$, where $u, v$, $w$ are arbitrary functions of $x, y, z$. The finite equations of the group are found by integrating

\footnotetext{
* Presented to the Society (Chicago) December 29, 1899, under a slightly different title. Received for publication January 13, 1900.
} 


$$
\frac{d x}{d t}=u(x, y, z), \quad \frac{d y}{d t}=v(x, y, z), \quad \frac{d z}{d t}=v(x, y, z),
$$

with the condition $x=a, y=b, z=c$ for $t=0$, if $a, b, c$ are the variables which are transformed into $x, y, z$ by the operations of the group. Thus $u$, $v, w$ are the values of the velocity-components at the point $x, y, z$.

Let $X, Y, Z$ denote the components of the force acting upon the unit of mass, $p$ the pressure, and $\rho$ the density at the point $x, y, z$. Then Euler's equations assume the form:

$$
\mathrm{K} u=X-\frac{1}{\rho} \partial p, \quad \mathrm{~K} v=Y-\frac{1}{\rho} \frac{\partial p}{\partial y}, \quad \mathrm{~K} w=Z-\frac{1}{\rho} \partial p
$$

and the equation of continuity becomes :

$$
\mathrm{K} \rho+\rho\left(\frac{\partial u}{\partial x}+\frac{\partial v}{\partial y}+\frac{\partial w}{\partial z}\right)=0 .
$$

Moreover $p$ and $\rho$ are connected by an equation :

$$
p=f(\rho),
$$

where the nature of the function $f(\rho)$ depends upon the character of the fluid considered.

Suppose that $u, v, w$ are arbitrarily assigned functions of $x, y, z$. Then by integrating (3) we get $x, y, z$ as functions of $a, b, c$, and $t$. The equations $(1)$, thus found, represent any one-parameter group $G$ in space. We can find the physical conditions corresponding to every group $G$. First $\rho$ may be found by integrating (4a). The simplest way to do this is to consider $\rho$ as a function of $a, b, c$ and $t$ rather than of $x, y, z$. For then, since the motion is steady, i. e., since $\partial \rho / \partial t=0, \mathrm{~K} \rho$ is simply $d \rho / d t$, and therefore $(4 a)$ may be written :

whence

$$
\frac{1 d \rho}{\rho} \frac{d \rho}{d t}=-\left(\frac{\partial u}{\partial x}+\frac{\partial v}{\partial y}+\frac{\partial w}{\partial z}\right)=-\sigma(a, b, c ; t)
$$

$$
\rho=\rho_{0} e^{-\int_{0}^{t} \sigma(a, b, c ; t) d t}
$$

where $\rho_{0}$ denotes the value of $\rho$ for $t=0$. In the case of steady motion, $\rho_{0}$ is not an arbitrary function of $a, b, c$ over any region of space. For if it were, $\rho$ would be the same function of $x, y, z$ that $\rho_{0}$ is of $a, b, c$ and therefore $\rho$ would be an arbitrary function of $x, y, z$ which according to $(4 a)$ is obviously not the case. The reason for this difficulty is that although we have assumed explicitly that $u, v, w$ are functions of $x, y, z$ alone, we have as yet made no explicit assumption of the same character about $\rho$, equation (5) being universally true whether the motion be stationary or not. 
If $S$ be a surface, cutting all of the lines of flow once and only once, $\rho$ can obviously be assigned arbitrary values for all points upon this surface. Of course $S$ may consist of several separate pieces. When any element of the fluid reaches this surface, its density must then, if the motion is stationary, assume the value corresponding to that point of $S$. Thus and only thus can the motion be stationary.

After $\rho$ is obtained $(4 b)$ gives $p$, and from (4), X, Y,Z can be found. If $u, v, w$ are real, and $\rho_{0}$ is positive, $\rho$ from (5) is positive as it should be. If $p$ should become negative, the fluid would tear.

In the case of an incompressible fluid, these results would be somewhat different : $p$ is not then a function of $\rho$. We then have

$$
\frac{\partial u}{\partial x}+\frac{\partial v}{\partial y}+\frac{\partial w}{\partial z}=0
$$

so that $u, v, w$ must be taken subject to this condition. The surfaces $\rho=$ constant are made up of lines of flow, for the density of an element of an incompressible fluid can not change. From one line of flow to another the density can vary according to an arbitrary law, for $(4 a)$ has reduced to $\mathrm{K} \rho=0$, which says only that the surfaces $\rho=$ constant are invariant under all transformations of the group, i. e., that they are made up of lines of flow, which we have already assumed to be the case. Since $p$ is not in general a function of $\rho$, in this case $p$ can be assumed to be an arbitrary function of $x, y, z$ and then $X, Y, Z$ are determined from (4).

If the fluid is homogeneous, without being incompressible, $\rho=$ constant, and then from $(4 a)$, since $\mathrm{K} \rho=0$, we get

$$
\frac{\partial u}{\partial x}+\frac{\partial v}{\partial y}+\frac{\partial w}{\partial z}=0
$$

i. e., although the fluid may not be incompressible, if it is homogeneous and has a steady motion, it moves just as an incompressible fluid of like density would. But if the fluid is not incompressible, $p$ is a certain function of $\rho$, and since $\rho=$ constant, $p=$ constant all through the fluid. The forces $X, Y, Z$ are again obtained from (4).

The one-parameter group, generated by $\mathrm{K} f$, has two families of invariant surfaces, viz., the $\infty^{2}$ surfaces :

$$
u_{1}(x, y, z)=\text { constant }, \quad u_{2}(x, y, z)=\text { constant }
$$

where $u_{1}, u_{2}$ are two independent solutions of $\mathrm{K} f=0$. The intersections of these surfaces are the lines of flow of the fluid.

Like all one-parameter groups, ours can be written in the form :

$$
\Omega_{i}(x, y, z)=\Omega_{i}(a, b, c)(i=1,2), \quad W(x, y, z)=W^{\top}(a, b, c)+t .
$$


Thus all steady fluid motions can be put into this form, and every set of equations of this kind represents a steady fuid motion.

It should be remarked, however, that it is not necessary that the functions $\Omega_{i}$ and $W$ should be represented by the same formula for all portions of space. Thus, we have more generally

$$
\begin{array}{lr}
\Omega_{k i}(x, y, z)=\Omega_{k i}(a, b, c) & (i=1,2), \\
W_{k i}(x, y, z)=W_{l i}(a, b, c)+t & (k=1,2,3, \cdots),
\end{array}
$$

the formulæ with index $k$ being valid only for a certain region $R_{k}$ of space.

Thus while the point $x, y, z$ is in $R_{1}, \Omega_{1 i}$ and $W_{1}$ are used, when it is in $R_{2}$, the next adjoining region, $\Omega_{2 i}$ and $W_{2}$ are employed, etc. Of course, for continuity, the values of $x, y, z$ obtained from the first set of equations must coincide with those found from the second set for all points upon the boundary between $R_{1}$ and $R_{2}$, i. e., $x, y, z$ must be continuous functions of $a, b, c, t$. The same is not necessary of $u, v, w$, i. e., in general the direction of the motion will undergo a discontinuous change on the bounding surface between two such regions. Such discontinuities will actually occur, for instance, if we consider the flow of a fluid in a channel whose walls are portions of analytical surfaces, which meet at angles different from $180^{\circ}$.

These considerations go a great way towards the solution of the general problem of the steady flow of a fluid in a channel bounded by any arbitrary surfaces whatsoever. An infinity of equations of the form (8) can be written down in every case, which fulfil the boundary conditions, and which represent a possible fluid motion. Among all these, it will be necessary to pick out those which correspond to the external forces at work, i. e., which give the values to $X, Y, Z$ belonging to that particular problem.

In most cases the forces $X, Y, Z$ have a potential, so that

$$
X=\frac{\partial V}{\partial x}, \quad Y=\frac{\partial V}{\partial y}, \quad Z=\frac{\partial V}{\partial z}
$$

In all such cases $u, v, w$ cannot be chosen at will, but are subject to the condition, that the values of $X, Y, Z$ computed from (4) can be written in the form (9). If we put

equations (4) become :

$$
P=\int \frac{d p}{\rho}
$$

$$
\mathrm{K} u=\frac{\partial(V-P)}{\partial \boldsymbol{x}}, \quad \mathrm{K} v=\frac{\partial(V-P)}{\partial y}, \quad \mathrm{~K} v=\frac{\partial(V-P)}{\partial z},
$$

and therefore, if the forces $X, Y, Z$ have a potential, 


$$
\mathrm{K} u d x+\mathrm{K} v d y+\mathrm{K} w d z=d(V-P)
$$

must be a complete differential. This is the condition which $u, v, w$ must verify in this case, or, what amounts to the same thing,

$$
\frac{\partial \mathrm{K} v}{\partial z}-\frac{\partial \mathrm{K} w}{\partial y}=0, \quad \frac{\partial \mathrm{K} w}{\partial x}-\frac{\partial \mathbf{K} u}{\partial z}=0, \quad \frac{\partial \mathbf{K} u}{\partial y}-\frac{\partial \mathbf{K} v}{\partial x}=0
$$

\section{§2. The fluid motion expressed by the general projective group.}

We shall confine ourselves to the case in which the forces have a potential and we shall find that the most general ternary projective group, which can express the steady motion of a fluid, if the forces have a potential, is a linear group.

For convenience in this paragraph we shall write $x_{1}, x_{2}, x_{3}, u_{1}, u_{2}, u_{3}$ instead of $x, y, z, u, v, w$.

The most general projective infinitesimal transformation is a linear combination of the following fifteen :

We can write it :

$$
p_{i}=\frac{\partial f}{\partial x_{i}}, \quad x_{i} p_{k}=T_{i k}, \quad x_{i} \sum_{k=1}^{3} x_{k} p_{k}=P_{i} \quad(i, k=1,2,3) .
$$

$$
\mathrm{K} f=\sum_{i=1}^{3} u_{i} \frac{\partial f}{\partial x_{i}}
$$

where

$$
u_{i}=\gamma_{i}+\sum_{k=1}^{3} c_{k i} x_{k}+x_{i} \sum_{k=1}^{3} c_{k} x_{k} \quad(i=1,2,3) .
$$

We must find the necessary and sufficient conditions in order that

may be a complete differential.

$$
\sum_{i=1}^{3} \mathrm{~K} u_{i} d x_{i}
$$

We obtain

$$
v_{i}=\mathrm{K} u_{i}=\left(c_{1} x_{1}+c_{2} x_{2}+c_{3} x_{3}\right) u_{i}+\sum_{k=1}^{3}\left(c_{k i}+c_{k i} c_{i}\right) u_{k} \quad(i=1,2,3) .
$$

But if the forces have a potential, equations (13) of $\S 1$ must be verified, the first of which is

We find

$$
\frac{\partial v_{3}}{\partial x_{2}}-\frac{\partial v_{2}}{\partial x_{3}}=0
$$

$$
\begin{aligned}
\frac{\partial v_{3}}{\partial x_{2}}-\frac{\partial v_{2}}{\partial x_{3}}=c_{2} u_{3}-c_{3} u_{2}+\left(c_{1} x_{1}+c_{2} x_{2}\right. & \left.+c_{3} x_{3}\right)\left(\frac{\partial u_{3}}{\partial x_{2}}-\frac{\partial u_{2}}{\partial x_{3}}\right) \\
& +\sum_{k=1}^{3}\left(\left(c_{k 3}+c_{k} x_{3}\right) \frac{\partial u_{k}}{\partial x_{2}}-\left(c_{k 2}+c_{k} x_{2}\right) \frac{\partial u_{k}}{\partial x_{3}}\right) .
\end{aligned}
$$


Now, from (2), we find

$$
\frac{\partial u_{i}}{\partial x_{j}}=c_{j i}+c_{j} x_{i}+\delta_{i j} \sum_{\lambda=1}^{3} c_{\lambda} x_{\lambda},
$$

where $\delta_{i j}=0$ for $i \neq j$, and $\delta_{i i}=1$.

Thus we obtain :

$$
\begin{aligned}
\frac{\partial v_{3}}{\partial x_{2}}- & \frac{\partial v_{2}}{\partial x_{3}}=c_{2} u_{3}-c_{3} u_{2}+\left[c_{23}-c_{32}+\left(c_{2} x_{3}-c_{3} x_{2}\right)\right] \sum_{\lambda=1}^{3} c_{\lambda} \dot{x_{\lambda}} \\
& \quad+\sum_{k=1}^{3}\left[\left(c_{k 3}+c_{k} x_{3}\right)\left(c_{2 k}+c_{2} x_{k}+\delta_{k 2} \sum_{\lambda=1}^{3} c_{\lambda} x_{\lambda}\right)-\left(c_{k 2}+c_{k} x_{2}\right)\left(c_{3 k}+c_{3} x_{k}+\delta_{k 3} \sum_{\lambda=1}^{3} c_{\lambda} x_{\lambda}\right)\right] .
\end{aligned}
$$

When this is arranged according to powers of $x_{1}, x_{2}, x_{3}$, the coefficient of each term must vanish. This gives the following equations, obtained by putting the coefficients of the quadratic terms equal to zero:

$$
c_{2} c_{3}=c_{3} c_{1}=c_{1} c_{2}=c_{2}^{2}-c_{3}^{2}=0,
$$

which gives at once $c_{2}=c_{3}=0$. Using one of the other equations (13) $\S 1$, we should find $c_{1}=0$ also.

Equating the other coefficients in $\left(\partial v_{2} / \partial x_{3}\right)-\left(\partial v_{3} / \partial x_{2}\right)$ to zero, we get only one other condition, and thus we have proved the theorem :

An arbitrary projective one-parameter group cannot represent the steady motion of a fluid under the influence of forces possessing a potential. To represent such a motion, the group must be a linear group. If

where

$$
\mathrm{K} f=\sum_{k=1}^{3} u_{i} \frac{\partial f}{\partial x_{i}}
$$

$$
u_{i}=\gamma_{i}+\sum_{k=1}^{3} c_{k i} x_{k}
$$

is the infinitesimal transformation of this linear group, the coefficients $c_{k i}$ must verify the three conditions :

$$
\left\{\begin{array}{l}
\sum_{k=1}^{3}\left(c_{k 3} c_{2 k}-c_{k 2} c_{3 k}\right)=0 \\
\sum_{k=1}^{3}\left(c_{k 1} c_{3 k}-c_{k 3} c_{1 k}\right)=0 \\
\sum_{k=1}^{3}\left(c_{k 2} c_{1 k}-c_{k 1} c_{2 k}\right)=0
\end{array}\right.
$$

which necessary conditions are also sufficient. 
§ 3. Fluid motion expressed by the ternary linear group.

In equations (18) we can put $\gamma_{i}=0$ without loss of generality. For the motions in which $\gamma_{i} \neq 0$ only differ from those for which $\gamma_{i}=0$ by a translation.

We assume then that the origin remains fixed during the entire motion, and we will resume the less symmetrical but more convenient notation, $x, y, z: u$, $v, w$ instead of $x_{1}, x_{2}, x_{3} ; u_{1}, u_{2}, u_{3}$. Let

$$
\left\{\begin{array}{l}
u=a_{1} x+b_{1} y+c_{1} z, \\
v=a_{2} x+b_{2} y+c_{2} z, \\
w=a_{3} x+b_{3} y+c_{3} z .
\end{array}\right.
$$

The components of the velocity of rotation of any element of the fluid are :

$$
\left\{\begin{array}{l}
\xi=\frac{1}{2}\left(\frac{\partial w}{\partial y}-\frac{\partial v}{\partial z}\right)=\frac{1}{2}\left(b_{3}-c_{2}\right), \\
\eta=\frac{1}{2}\left(\frac{\partial u}{\partial z}-\frac{\partial w}{\partial x}\right)=\frac{1}{2}\left(c_{1}-a_{3}\right), \\
\zeta=\frac{1}{2}\left(\frac{\partial v}{\partial x}-\frac{\partial u}{\partial y}\right)=\frac{1}{2}\left(a_{2}-b_{1}\right),
\end{array}\right.
$$

so that $\xi, \eta, \zeta$ are the same for all points of the fluid. Hence at all points the axis of rotation has the same direction, and the angular velocity of rotation is the same, viz.:

$$
\omega=\sqrt{\xi^{2}+\eta^{2}+\zeta^{2}}
$$

Let us take our $z$ axis parallel to this direction. Then

$$
\xi=\eta=0, \quad \zeta=\omega,
$$

and hence, for this choice of coördinates,

$$
b_{3}=c_{2}, \quad c_{1}=a_{3}, \quad a_{2}=b_{1}+2 \omega,
$$

so that we can write more simply

$$
\left\{\begin{array}{crlrl}
u & = & A x+(H-\omega) y+G z, \\
v & = & (H+\omega) x+ & B y+F z, \\
w & = & G x+ & F y+C z .
\end{array}\right.
$$

If the forces have a potential, equations (19) give

$$
(A+B) \omega=0, \quad F \omega=0, \quad G \omega=0 .
$$

Therefore, either $\omega=0$, or

$$
A+B=0, \quad F=0, \quad G=0 .
$$


If $\omega=0$, we have a velocity potential

$$
\phi=\frac{1}{2}\left(A x^{2}+B y^{2}+C z^{2}+2 F y z+2 G x z+2 H x y\right),
$$

so that the lines of flow are the orthogonal trajectories of the system of similar surfaces of the second order:

$$
\phi=\text { constant. }
$$

In the second case $(21)$ reduces to

$$
\left\{\begin{array}{l}
u=A x+(H-\omega) y, \\
v=(H+\omega) x-A y, \\
w=r z .
\end{array}\right.
$$

The motion can be decomposed into a rotation and a motion with a velocity potential. But it is simpler to treat it without so decomposing it. This case again separates into two distinct sub-cases.

In the first sub-case,

Then, if

$$
A^{2}+H^{2}-\omega \neq 0
$$

$$
\left\{\begin{array}{l}
\rho_{1}=+\sqrt{A^{2}+H^{2}-\omega}=\rho, \quad \rho_{2}=-\rho_{1}=-\rho, \\
\lambda_{i}=-\frac{H-\omega}{A-\rho_{i}}=\frac{A+\rho_{i}}{H+\omega},
\end{array}\right.
$$

the finite equations of the group can be written as follows:

$$
x-\lambda_{1} y=e^{-\rho t}\left(a-\lambda_{1} b\right), \quad x-\lambda_{2} y=e^{+\rho t}\left(a-\lambda_{2} b\right), \quad z=c e^{C t},
$$

where $a, b, c$ are the values of $x, y, \approx$ for $t=0$.

The surfaces :

$$
z^{-\rho}\left(x-\lambda_{1} y\right)^{-C}=\text { constant }, \quad z^{\rho}\left(x-\lambda_{2} y\right)^{-C}=\text { constant },
$$

which are real or imaginary cylinders, are therefore invariant. Their real intersections are the lines of flow.

The function

$$
\left(x-\lambda_{1} y\right)\left(x-\lambda_{2} y\right)
$$

is also invariant. Therefore the projections of the lines of flow upon the $x y$. plane are the similar conics :

$$
x^{2}-\frac{2 A}{H+\omega} x y+\frac{\omega-H}{\omega+H} y^{2}=\text { constant }
$$

i. e., the lines of flow are situated upon a cylinder of the second order, whose elements are parallel to the $z$ axis. 
The physical conditions, corresponding to this motion, are obtained from the considerations of $\S 1$. We have, in particular, from (11),

$$
V-p=\frac{1}{2}\left[\left(A^{2}+H^{2}-\omega\right)\left(x^{2}+y^{2}\right)+c^{2} z^{2}\right]+\text { constant. }
$$

The motion, here considered, becomes periodic if $\rho$ is purely imaginary, and $c=0$, as may be seen by solving (24) for $x, y, z$. The lines of flow are then similar ellipses in parallel planes. It can be shown that this motion is possible for an incompressible homogeneous fluid filling an ellipsoidal space, the particles attracting each other according to Newton's law. This has been studied in detail by Dirichlet and Dedekind.* It can also be easily shown that the analogous result is true for an elliptic cylinder of infinite length, provided that $H$ and $\omega$ satisfy the equation :

$$
\left(H^{2}-\omega^{2}\right) \frac{H^{2}+4}{\pi f \rho}+4=0,
$$

where $\rho$ is the density, and $f$ a numerical constant, depending upon the units employed. If $a^{\prime}$ and $b^{\prime}$ are the semi-axes of the elliptic section of the cylinder their ratio will be obtained from the equation:

In the second sub-case,

$$
\frac{a^{\prime}}{\bar{b}^{\prime}}=\sqrt{\frac{\omega-H}{\omega+H}} .
$$

$$
\left\{\begin{array}{l}
A^{2}+H^{2}-\omega=0 \\
\lambda=-\frac{H-\omega}{A}=\frac{A}{H+\omega} .
\end{array}\right.
$$

The finite equations of the group can be written :

$$
x-\lambda y=a-\lambda b, \quad \frac{y}{x-\lambda y}=\frac{b}{a-\lambda b}+(H+\omega) t, \quad z=c e^{c t} .
$$

If we chose the plane $x-\lambda y=0$ as $x z$-plane, and the plane $\lambda x+y=0$ as $y z-$ plane, the planes $y=$ constant are invariant, and the lines of flow are the curves :

$$
z=\text { constant } e^{\frac{C}{2 \omega} \frac{x}{y}}, \quad y=\text { constant }
$$

i. e., exponential curves in these planes.

\section{\$4. Fluid motion expressed by a linearoid group.}

Let us consider next a one-parameter group, generated by

$$
\mathrm{K} f=u \frac{\partial f}{\partial x}+v \frac{\partial f}{\partial y}+w \frac{\partial f}{\partial z},
$$

* Crelle's Journal, vol. 58. 
where

$$
\left\{\begin{array}{l}
u=\phi_{1} x+\phi_{2} y+\phi_{3}, \\
v=\psi_{1} x+\psi_{2} y+\psi_{3}, \\
w=\chi_{1} x+\chi_{2} y+\chi_{3},
\end{array}\right.
$$

$\phi_{1}, \cdots, \chi_{3}$ being functions of $z$ only. It will be of the class called linearoid by me in another connection. Then,

$$
\left\{\begin{array}{r}
\mathrm{K} u=\left(\phi_{1}^{2}+\phi_{2} \psi_{1}+\phi_{3}^{\prime} \chi_{1}\right) x+\left(\phi_{1} \phi_{2}+\phi_{2} \psi_{2}+\phi_{3}^{\prime} \chi_{2}\right) y+\phi_{1} \phi_{3}+\phi_{2} \psi_{3} \\
+\phi_{3}^{\prime} \chi_{3}+\phi_{1}^{\prime} \chi_{1} x^{2}+\left(\phi_{1}^{\prime} \chi_{2}+\phi_{2}^{\prime} \chi_{1}\right) x y+\phi_{2}^{\prime} \chi_{2} y^{2} \\
\mathrm{~K} v=\left(\psi_{1} \phi_{1}+\psi_{2} \psi_{1}+\psi_{3}^{\prime} \chi_{1}\right) x+\left(\psi_{1} \phi_{2}+\psi_{2}^{2}+\psi_{3}^{\prime} \chi_{2}\right) y+\psi_{1} \phi_{3}+\psi_{2} \psi_{3} \\
+\psi_{3}^{\prime} \chi_{3}+\psi_{1}^{\prime} \chi_{1} x^{2}+\left(\psi_{1}^{\prime} \chi_{2}+\psi_{2}^{\prime} \chi_{1}\right) x y+\psi_{2}^{\prime} \chi_{2} y^{2}, \\
\mathrm{~K} w=\left(\chi_{1} \phi_{1}+\chi_{2} \psi_{1}+\chi_{3}^{\prime} \chi_{1}\right) x+\left(\chi_{1} \phi_{2}+\chi_{2} \psi_{2}+\chi_{3}^{\prime} \chi_{2}\right) y+\chi_{1} \phi_{3}+\chi_{2} \psi_{3} \\
+\chi_{3}^{\prime} \chi_{3}+\chi_{1}^{\prime} \chi_{1} x^{2}+\left(\chi_{1}^{\prime} \chi_{2}^{\prime}+\chi_{2}^{\prime} \chi_{1}\right) x y+\chi_{2}^{\prime} \chi_{2} y_{2},
\end{array}\right.
$$

where the accents denote derivatives with respect to $z$.

Write this as follows :

$$
\left\{\begin{array}{l}
\mathrm{K} u=\lambda_{1} x^{2}+2 \mu_{1} x y+\nu_{1} y^{2}+2 \rho_{1} x+2 \sigma_{1} y+2 \tau_{1}, \\
\mathrm{~K} v=\lambda_{2} x^{2}+2 \mu_{2} x y+\nu_{2} y^{2}+2 \rho_{2} x+2 \sigma_{2} y+2 \tau_{2} \\
\mathrm{~K} \dot{w}=\lambda_{3} x^{2}+2 \mu_{3} x y+\nu_{3} y^{2}+2 \rho_{3} x+2 \sigma_{3} y+2 \tau_{3}
\end{array} ;\right.
$$

the conditions for the integrability of

$$
\mathrm{K} u \cdot d x+\mathrm{K} v \cdot d y+\mathbf{K} w \cdot d z,
$$

i. e., for the existence of a potential for the corresponding fluid motion, become :

Hence

$$
\left\{\begin{array}{lll}
\mu_{1}-\lambda_{2}=0, & \nu_{1}-\mu_{2}=0, & \sigma_{1}-\rho_{2}=0, \\
\rho_{2}^{\prime}-\mu_{3}=0, & \sigma_{2}^{\prime}-\nu_{3}=0, & \tau_{2}^{\prime}-\sigma_{3}=0, \\
\rho_{1}^{\prime}-\lambda_{3}=0, & \sigma_{1}^{\prime}-\mu_{3}=0, & \tau_{1}^{\prime}-\rho_{3}=0, \\
\lambda_{1}^{\prime}=\mu_{1}^{\prime}=\nu_{1}^{\prime}=\lambda_{2}^{\prime}=\mu_{2}^{\prime}=\nu_{2}^{\prime}=0 . &
\end{array}\right.
$$

$$
\left\{\begin{array}{l}
2\left(\mu_{1}-\lambda_{2}\right)=\left(\phi_{2}^{\prime}-2 \psi_{1}^{\prime}\right) \chi_{1}+\phi_{1}^{\prime} \chi_{2}=0, \\
2\left(\nu_{1}-\mu_{2}\right)=-\psi_{2}^{\prime} \chi_{1}+\left(2 \phi_{2}^{\prime}-\psi_{1}^{\prime}\right) \chi_{2}=0,
\end{array}\right.
$$

so that we have two cases to distinguish, according as $\chi_{1}=\chi_{2}=0$, or the determinant

$$
\left(\phi_{2}^{\prime}-2 \psi_{1}^{\prime}\right)\left(2 \phi_{2}^{\prime}-\psi_{1}^{\prime}\right)+\phi_{1}^{\prime} \psi_{2}^{\prime}=0
$$


Let us consider first case I, in which $\chi_{1}=\chi_{2}=0$. Then we find from the definition of these quantities,

$$
\lambda_{1}=\mu_{1}=\nu_{1}=\lambda_{2}=\mu_{2}=\nu_{2}=\lambda_{3}=\mu_{3}=\nu_{3}=\rho_{3}=\sigma_{3}=0,
$$

and from $(30)$,

$$
\rho_{1}^{\prime}=\rho_{2}^{\prime}=\sigma_{1}^{\prime}=\sigma_{2}^{\prime}=\tau_{1}^{\prime}=\tau_{2}^{\prime}=0,
$$

so that $\rho_{1}, \rho_{2}, \sigma_{1}, \sigma_{2}, \tau_{1}, \tau_{2}$ are constants.

Now since $\chi_{1}=\chi_{2}=0$, we have

$$
\begin{cases}2 \rho_{1}=\phi_{1}^{2}+\phi_{2} \psi_{1}, & 2 \rho_{2}=\psi_{1}\left(\phi_{1}+\psi_{2}\right), \\ 2 \sigma_{1}=\phi_{2}\left(\phi_{1}+\psi_{2}\right), & 2 \sigma_{2}=\phi_{2} \psi_{1}+\psi_{2}^{2} .\end{cases}
$$

The only further condition is $\sigma_{1}=\rho_{2}$, whence either

$$
\phi_{1}+\psi_{2}=0, \quad \text { or } \quad \phi_{2}=\psi_{1} .
$$

We will first assume that $\phi_{1}+\psi_{2}=0$, and call this case I $a$. Then we have, in this case,

$$
\sigma_{1}=\rho_{2}=0, \quad \phi_{1}^{2}+\phi_{2} \psi_{1}=2 \rho_{1}=2 \sigma_{2}=2 \sigma,
$$

where $\sigma$ is a constant. Thus in case $\mathrm{I} a$ the functions $\chi_{1}$ and $\chi_{2}$ vanish, and between the other functions we have the relations :

$$
\left\{\begin{array}{l}
\phi_{1}+\psi_{2}=0, \quad \phi_{1}^{2}+\phi_{2} \psi_{1}=2 \sigma=\text { constant } \\
\phi_{1} \phi_{3}+\phi_{2} \psi_{3}+\chi_{3} \phi_{3}^{\prime}=2 \tau_{1} \\
\psi_{1} \phi_{3}+\psi_{2} \psi_{3}+\chi_{3} \psi_{3}^{\prime}=2 \tau_{2}
\end{array}\right.
$$

where $\tau_{1}, \tau_{2}$ are also constants, and where $\phi_{1}, \phi_{2}, \chi_{3}$ may be taken as arbitrary functions of $z$.

In the second case, case $\mathrm{I} b$, we have

whence

$$
\phi_{2}=\psi_{1},
$$

$$
\left\{\begin{array}{l}
2 \rho_{2}=2 \sigma_{1}=\phi_{2}\left(\phi_{1}+\psi_{2}\right), \\
2 \rho_{1}=\phi_{2}^{2}+\phi_{2}^{2}, \quad 2 \sigma_{2}=\phi_{2}^{2}+\psi_{2}^{2},
\end{array}\right.
$$

where $\phi_{1}$ and $\psi_{2}$ may be taken to be arbitrary functions of $z$, and the equations for $\phi_{3}, \psi_{3}, \chi_{3}$ are of the same form as in (34).

If the fluid is incompressible we have further, $\chi_{3}=$ constant in case $\mathrm{I} a$, and in case $\mathrm{I} b$, we find

$$
\chi_{3}=-\int\left(\phi_{1}+\psi_{2}\right) d z+\text { constant }
$$


as is seen at once from the condition of incompressibility, viz. :

$$
\frac{\partial u}{\partial x}+\frac{\partial v}{\partial y}+\frac{\partial w}{\partial z}=0
$$

We shall not at present further discuss the fluid motions thus analytically determined. The group theoretical considerations have enabled us to discover a considerable class of fluid motions, whose entire theory depends only on linear differential equations.

Let us now carry out the corresponding investigation for case II, in which $\chi_{1}$ and $\chi_{2}$ do not both vanish. We have then

$$
\left(\phi_{2}^{\prime}-2 \psi_{1}^{\prime}\right)\left(2 \phi_{2}^{\prime}-\psi_{1}^{\prime}\right)+\phi_{1}^{\prime} \psi_{2}^{\prime}=0
$$

and further, as before (equations (30)) $\lambda_{1}, \mu_{1}, \nu_{1}, \lambda_{2}, \mu_{2}, \nu_{2}$ are constants. By the definition of these quantities, we have

Therefore and hence

$$
\left\{\begin{array}{lll}
\phi_{1}^{\prime} \chi_{1}=\lambda_{1}, & \phi_{2}^{\prime} \chi_{1}+\phi_{1}^{\prime} \chi_{2}=2 \mu_{1}, & \phi_{2}^{\prime} \chi_{2}=\nu_{1}, \\
\psi_{1}^{\prime} \chi_{1}=\lambda_{2}, & \psi_{2}^{\prime} \chi_{1}+\psi_{1}^{\prime} \chi_{2}=2 \mu_{2}, & \psi_{2}^{\prime} \chi_{2}=\nu_{2}
\end{array}\right.
$$

where $a$ and $\beta$ denote the ratios $\lambda_{1}: \nu_{1}$ and $2 \mu_{1}: \nu_{1}$.

Multiplying (32) by $\chi_{1}^{2} \chi_{2}^{2}$, and using (36) and (38), we obtain a third quadratic for $\chi_{1}$ and $\chi_{2}$, viz. :

so that also

$$
\nu_{i} \chi_{1}^{2}+\lambda_{i} \chi_{2}^{2}-2 \mu_{i} \chi_{1} \chi_{2}=0 \quad(i=1,2),
$$

$$
\lambda_{1}: 2 \mu_{1}: \nu_{1}=\lambda_{2}: 2 \mu_{2}: \nu_{2}=a: \beta: 1
$$

Now if we rotate the $x$ axis through an angle $\theta$, the form of $u, v, w$ is not changed, and $w$ becomes

$$
\begin{aligned}
& \quad \chi_{1}(x \cos \theta-y \sin \theta)+\chi_{2}(x \sin \theta+y \cos \theta)+\chi_{3} \\
& \quad=\left(\chi_{1} \cos \theta+\chi_{2} \sin \theta\right) x+\left(-\chi_{1} \sin \theta+\chi_{2} \cos \theta\right) y+\chi_{3} .
\end{aligned}
$$

If we put

$$
\tan \theta=\frac{\nu_{1}}{\lambda_{2}}
$$

according to (39), the coefficient of $y$ in the transformed $w$ will vanish. 
We can therefore assume from the beginning, without loss of generality, that $\chi_{2}=0$. Having already treated the case $\chi_{1}=\chi_{2}=0$, we will now assume $\chi_{1} \neq 0, \chi_{2}=0$.

From (36) we have, in this case,

$$
\begin{cases}\phi_{1}^{\prime}=\frac{\lambda_{1}}{\chi_{1}}, & \phi_{2}^{\prime}=\frac{2 \mu_{1}}{\chi_{1}}, \quad \nu_{1}=0, \\ \psi_{1}^{\prime}=\frac{\lambda_{2}}{\chi_{1}}, & \psi_{2}^{\prime}=\frac{2 \mu_{2}}{\chi_{1}}, \quad \nu_{2}=0 .\end{cases}
$$

Since $\nu_{2}=0,(30)$ gives $\mu_{2}=0$. Therefore, if

we have, since $\mu_{1}=\lambda_{2}$,

$$
\int \frac{d z}{\chi_{1}}=\phi
$$

$$
\begin{cases}\phi_{1}=\lambda_{1} \phi+a, & \phi_{2}=2 \lambda_{2} \phi+\beta, \\ \psi_{1}=\lambda_{2} \phi+\gamma, & \psi_{2}=\delta,\end{cases}
$$

where $a, \beta, \gamma, \delta$ denote four arbitrary constants.

We find further,

$$
\lambda_{3}=\chi_{1}^{\prime} \chi_{1}, \quad \mu_{3}=0, \quad \nu_{3}=0 .
$$

Therefore, from (30), we notice that $\rho_{2}, \sigma_{2}, \sigma_{1}$ are constants. Now we have, with the values for $\phi_{1}, \phi_{2}, \psi_{1}, \psi_{2}$ just found,

$$
2 \sigma_{2}=2 \lambda_{2}^{2} \phi^{2}+\lambda_{2}(\beta+2 \gamma) \phi+\beta \gamma+\delta^{2},
$$

so that $\lambda_{2}$ must vanish. Then

$$
2 \sigma_{1}=\beta\left(\lambda_{1} \phi+a+\delta\right),
$$

so that $\lambda_{1}$ must also vanish. From the definition of $\rho_{2}$, which must be a constant, follows :

$$
\psi_{3}^{\prime}=\frac{2 \rho_{2}-\gamma(a+\delta)}{\chi_{1}}=\frac{(\beta-\gamma)(a+\delta)}{\chi_{1}},
$$

since $\rho_{2}=\sigma_{1}$. Similarly we find, from $\rho_{1}^{\prime}=\lambda_{3}=\chi_{1}^{\prime} \chi_{1}$,

$$
\phi_{3}^{\prime}=\chi_{1}+\frac{\epsilon-a^{2}-\beta \gamma}{\chi_{1}},
$$

where $\epsilon$ denotes another constant.

Substituting all these values into the only two equations (30) not yet used, viz. : $\tau_{2}^{\prime}=\sigma_{3}, \tau_{1}^{\prime}=\rho_{3}$, we find the following conditions for $\chi_{1}$ and $\chi_{3}$ : 


$$
\left\{\begin{array}{r}
(\gamma-\beta) \chi_{1}+\frac{\gamma\left(\epsilon-a^{2}-\beta \gamma\right)+\delta(\beta-\gamma)(a+\delta)}{\chi_{1}}+(\beta-\gamma)(a+\delta) \frac{d}{d z}\left(\frac{\chi_{3}}{\chi_{1}}\right)=0 \\
\frac{a\left(\epsilon-a^{2}-\beta \gamma\right)+\beta(\beta-\gamma)(a-\delta)}{\chi_{1}}+\frac{d}{d z}\left(\chi_{1} \chi_{3}\right) \\
+\left(\epsilon-a^{2}-\beta \gamma\right) \frac{d}{d z}\left(\frac{\chi_{3}}{\chi_{1}}\right)-\chi_{1} \chi_{3}^{\prime}=0 .
\end{array}\right.
$$

The fluid motion, in this case, is therefore given by the equations :

$$
\left\{\begin{array}{l}
u=a x+\beta y+\phi_{3}, \\
v=\gamma x+\delta y+\psi_{3}, \\
w=\chi_{1} x+\chi_{3},
\end{array}\right.
$$

where $a, \beta, \gamma, \delta$ are constants, where $\chi_{1}$ and $\chi_{3}$ are obtained as functions of $z$ by integrating (43), and where $\phi_{3}$ and $\psi_{3}$ are determined from (41) and (42).

If the fluid is incompressible, we have

i. e.,

$$
\frac{\partial u}{\partial x}+\frac{\partial v}{\partial y}+\frac{\partial w}{\partial z}=a+\delta+\chi_{1}^{\prime} x+\chi_{3}^{\prime}=0
$$

$$
\chi_{1}=\eta, \quad \chi_{3}=-(a+\delta) z+\theta,
$$

where $\eta$ and $\theta$ are constants. Moreover $\theta$ can be put equal to zero, if the $x y$ plane be appropriately chosen. Substituting these values in (43), we get relations between $\eta$ and the other constants. But as will be seen from (41) and (42) the group in this case becomes a ternary linear one, all of the coefficients being constants.

Thus Case II of this paragraph gives nothing new for incompressible fluids.

The detailed discussion of the fluid motions here determined, as well as those of the preceding case, will be left for a future occasion.

University of California, Berkeley. 